Chirurg 2014 · 85:472-473

DOI 10.1007/s00104-013-2665-y

Online publiziert: 21. Mai 2014

(c) Springer-Verlag Berlin Heidelberg 2014

\section{H. Dralle}

Universitätsklinik für Allgemein-, Viszeral- und Gefäßchirurgie,

Universitätsklinikum Halle, Medizinische Fakultät, Halle (Saale)

\title{
Molekulare Bildgebung in der onkologischen Chirurgie
}

Die molekulare Bildgebung hat sich in den zurückliegenden Jahren zu einem nahezu unverzichtbaren Bestandteil auch der onkologischen Chirurgie entwickelt. Mithilfe der kombinierten Schichtbildverfahren (Computertomographie [CT], neuerdings Magnetresonanztomographie [MRT]) ist eine Fusion struktureller und stoffwechseltypischer Merkmale, d. h. aus onkologischer Sicht nicht nur eine Lokalisation, sondern zugleich eine Momentaufnahme der Aktivität bzw. Differenzierung und im Verlauf eine Abschätzung der Dynamik von Tumoren, möglich. Hauptindikationen zur positronenemissionstomographischen (PET-)Untersuchung sind daher die Charakterisierung einer tumorverdächtigen Läsion (z. B. Lungenrundherd unklarer Dignität), die Tumorsuche bei erhöhten Tumormarkern oder beim CUP („cancer of unknown primary origin“) -Syndrom, das Tumorstaging zur Therapiestratifizierung, das Therapiemonitoring unter Chemotherapie und die postoperative Verlaufskontrolle bei Metastasenverdacht (Beitrag J. Kotzer$k e)$. Einsatz und Interpretation der PETUntersuchung erfordern aus chirurgischer Sicht nicht nur Kenntnisse der Grenzen der PET-Technik (falsch-positive/falschnegative Befunde), sondern auch Grundkenntnisse hinsichtlich der in Abhängigkeit von der Fragestellung zu wählenden Radiopharmaka. Aufgrund gegenwärtiger Studien ist davon auszugehen, dass durch die Hybridbildgebung ein diagnostischer Zugewinn in $40 \%$ möglich ist und sich in etwa einem Drittel der Fälle eine Änderung des therapeutischen Regimes ergibt.

\section{1) Bei ca. 40\% der \\ Patienten ergibt sich ein diagnostischer Zugewinn}

Die in diesem Heft von Der Chirurg unter dem Schwerpunktthema „Molekulare Bildgebung in der onkologische Chirurgie" diskutierten Hauptfragen waren daher die aktuelle Bedeutung der PET-Technik bei der

- Rezidiv- und Metastasendarstellung,

- Beurteilung des Therapieansprechens unter neoadjuvanter (Radio)Chemotherapie,

- Therapie mit peptidvermittelten Radiorezeptoren (PRRT).

Im Rahmen der viszeralchirurgischen Onkologie hat die molekulare Bildgebung Bedeutung im Rahmen des Stagings bzw. der Rezidiv- und Metastasendarstellung vor allem beim Schilddrüsenkarzinom (C. Haane und M. Colombo-Benkmann), Rektumkarzinom (A. Wiegering und Mitarbeiter), gastrointestinalen Stromatumor (GIST; U. Ronellenfitsch und Mitarbeiter) und neuroendokrinen Tumoren (Homman und Mitarbeiter) erlangt, beim Ösophaguskarzinom (K. Ott und Mitarbeiter), Rektumkarzinom und GIST-Tumoren steht die Frage des Therapieansprechens unter neoadjuvanter (Radio-)Chemotherapie im Vordergrund; zur Planung einer peptidvermittelten Radiorezeptortherapie wird die PET-Technik bislang lediglich bei metastasierten neuroendokrinen Karzinomen eingesetzt.

Beim Schilddrüsenkarzinom hat die Fluordesoxyglucose(FDG)-PET/CT einen mittlerweile fest etablierten Stellenwert in der Rezidiv- und Metastasendiagnostik beim prognostisch ungünstigen radiojodrefraktären differenzierten Karzinom. Die frühzeitige Erkennung und chirurgische Therapie lokoregionär ohne PET nicht lokalisierbarer Rezidive ist prognostisch relevant. Auch beim medullären Karzinom kann durch geeignete Radiopharmakaauswahl (FDG oder DOPA) die PET einen Hinweis auf den Differenzierungsgrad der Metastasen und damit auf die Prognose geben.

Beim neoadjuvant therapierten Ösophaguskarzinom korreliert der SUV (,standard uptake value") mit dem Therapieansprechen, die Ergebnisse sind jedoch bislang nicht hinreichend gesichert, um im therapeutischen Konzept berücksichtigt zu werden.

Beim Rektumkarzinom dient die PET vor allem dem lokoregionären und hepatischen Rezidivnachweis bei unklaren Raumforderungen oder lediglich Erhöhung des karzinoembryonalen Antigens. Auch bei diesem Karzinom korreliert der SUV mit der Prognose und dem Ansprechen auf eine neoadjuvante Therapie, insbesondere die hierfür zu setzenden Kriterien (SUV-Cut-off-Werte; bester Zeitpunkt der PET) sind jedoch noch nicht 
klar, um therapeutisch relevant umgesetzt zu werden.

Auch bei GIST-Tumoren kann die PET wichtige Hinweise für das Ansprechen unter Therapie mit Thyrosinkinaseinhibitoren liefern, da Metastasen von GIST-Tumoren eine nicht selten diskordante Progression entwickeln. Die ge nauen Kriterien für eine PET-basierte Therapiemodifikation sind jedoch bislang nicht standardisiert, sodass auch bei diesen Tumoren der therapeutische Stellenwert der molekularen Bildgebung noch nicht abschließend zu bewerten ist.

Metastasierte neuroendokrine Karzinome (NEC) nehmen bez. der PET insofern einen besonderen Stellenwert ein, als dass die PET/CT mit ${ }^{68} \mathrm{Ga}$-markierten Somatostatinanaloga nicht nur sehr effektiv zur Metastasendiagnostik gut differenzierter NEC eingesetzt werden kann, sondern auch verifiziert, inwieweit ein therapeutischer Einsatz im Rahmen der Radiorezeptortherapie im Einzelfall möglich ist.
Fazit: PET 2014 bedeutet aus viszeralchirurgisch-onkologischer Sicht einen ganz wesentlichen Zugewinn für die auch chirurgisch relevante Rezidiv- und Metastasendiagnostik. Für das Therapieansprechen unter neoadjuvanter Therapie ergeben sich jedoch trotz der Vorteile der PET (Quantifizierbarkeit des Tumormetabolismus) und der PET/CT/MRT (genaue Lokalisation, Größenbestimmung) und deutlicher Hinweise für eine SUV-bezogene prognostische Relevanz derzeit noch keine gesicherten Ansatzpunkte für eine PET-bezogene Therapiestratifizierung.
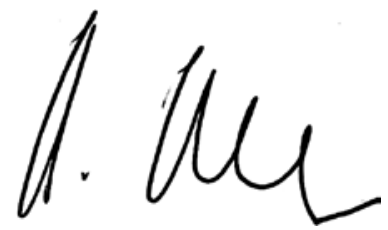

Prof. Dr. Dr. h.c. H. Dralle

\section{Korrespondenzadresse}

Prof. Dr. Dr. h.c. H. Dralle

Universitätsklinik für Allgemein-, Viszeralund Gefäßchirurgie, Universitätsklinikum Halle, Medizinische Fakultät,

Ernst-Grube-Str. 40, 06097 Halle (Saale)

henning.dralle@uk-halle.de

Interessenkonflikt. H. Dralle gibt an, dass kein Interessenkonflikt besteht.

\section{e.Curriculum Geriatrie: Modul 3 ist online!}

Vertiefen Sie in der Springer Medizin e.Akademie Ihr geriatrisches Fachwissen.

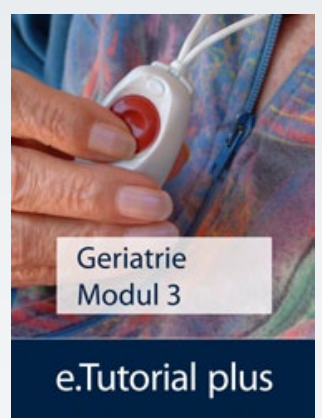

Das Modul 3

"Notfallmedizin im Alter"

ist:

- mit 5 CME-Punkten zertifiziert,

- interaktiv und multimedial,

- praxisnah mit zahlreichen

Fallbeispielen und Empfehlungen, Ein Kooperationsprojekt mit:

- kostenfrei!
Punkten Sie jetzt kostenfrei in der e.Akademie!

Sie finden diese und weitere Fortbildungsmodule im

e.Curriculum Geriatrie unter:

www.springermedizin.de/ecurriculum-geriatrie

Robert Bosch Stiftung $\rightarrow$ DGG

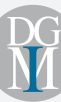

